\title{
Correction to: Requirements Engineering: Foundation for Software Quality
}

\author{
Nazim Madhavji, Liliana Pasquale, Alessio Ferrari (D), \\ and Stefania Gnesi
}

\section{Correction to: \\ N. Madhavji et al. (Eds.): Requirements Engineering: \\ Foundation for Software Quality, LNCS 12045, https://doi.org/10.1007/978-3-030-44429-7}

The book was inadvertently published with only two volume editors "Nazim Madhavji and Liliana Pasquale" whereas there should have been four "Nazim Madhavji, Liliana Pasquale, Alessio Ferrari and Stefania Gnesi”. The missing two volume editors were added in the book and the source line was updated accordingly. 